\title{
TSH SECRETING PITUITARY ADENOMA: A CASE REPORT
}

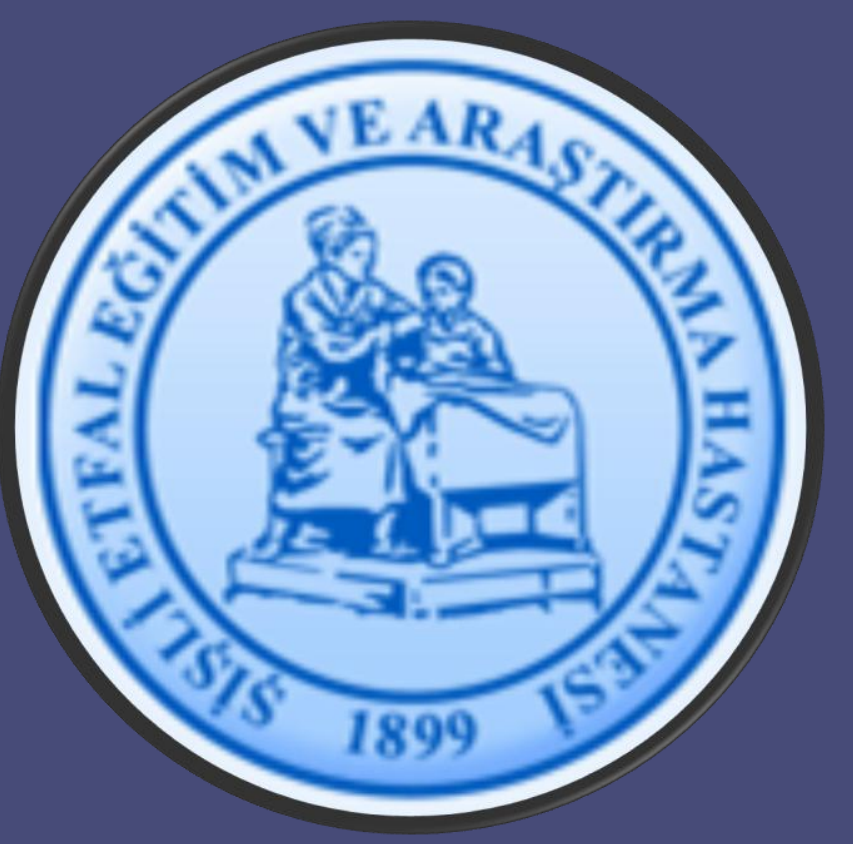

\author{
Feyza Yener Ozturk, Selvinaz Erol, Savas Karatas, \\ Muhammed Masum Canat, Idris Kuzu, Yuksel Altuntas \\ Sisli Etfal Training and Research Hospital \\ Department of Endocrinology and Metabolism, Istanbul-TURKEY
}

\section{INTRODUCTION}

Thyrotropin secreting pituitary adenomas are rare, constituting less than $2 \%$ of pituitary adenomas. Thirty percent of these tumors may be plurihormonal. Most common cosecreted hormone is $\mathrm{GH}$ and the least one is PRL. We report here a case of plurihormonal pituitary adenoma symptomatic for TSH secretion.

\section{CASE REPORT}

A 35 year old female admitted to hospital because of fatigue, heat intolerance, headache, galactorrhea and menstrual irregularity. Her laboratory analysis showed hyperprolactinemia (PRL:74 ng/ml), and high fT3 level with inappropriately normal TSH. TSH:3,14 ulU/mL (N:0,27-4,2 $\mathrm{ulU} / \mathrm{ml}) \quad \mathrm{fT} 3: 4,67 \mathrm{pg} / \mathrm{ml} \quad(\mathrm{N}: 1.96-4.36), \quad \mathrm{fT} 4: 1,24 \mathrm{ng} / \mathrm{ml}$ ( $\mathrm{N}: 0,72-1,56 \mathrm{ng} / \mathrm{dl})$. Growth hormone level was also high but IGF-1 levels were all normal and she didn't have any signs or symptoms of acromegaly. Pituitary MRI showed $18 \times 15 \mathrm{~mm}$ macroadenoma extending to suprasellar region. She was followed-up with cabergolin for a year. Then she had transsphenoidal hypophysectomy. The immunohistochemical staining showed that tumor cells were strongly reactive to $\mathrm{GH}, \mathrm{PRL}$ and TSH; Ki67 index was 2\%. Four months after operation, she got pregnant. There was no biochemical abnormality during pregnancy. Her pituitary MRI didn't show any residuel image. But at postpartum 2 nd month, high fT3 and fT4 levels were revealed. PRL was $60 \mathrm{ng} / \mathrm{dl}$ (analysis was done 4 hours after breastfeeding), FT3:4.39 pg/ml, FT4:1,66 $\mathrm{ng} / \mathrm{dl}, \mathrm{TSH}: 2,33 \mathrm{ulU} / \mathrm{mL}, \mathrm{GH}: 2,09 \mathrm{ng} / \mathrm{ml}, \mathrm{IGF}-1: 205 \mathrm{ng} / \mathrm{ml}$ (N:109-284), cortisol was suppressed after dexamethasone suppression test $(1,57 \mathrm{mcg} / \mathrm{dl})$. Physical examination revealed only tachycardia. Thyroid autoantibodies were all negative. Thyroid USG was normal other than a 5,3 $\mathrm{mm}$ heterogenous, isoechoic nodule. SHBG was normal but glycoprotein alpha subunit to TSH molar ratio was high. TSH levels failed to increase after TRH stimulation test. A pituitary adenoma $12 \times 11 \mathrm{~mm}$ in diameter compressing the optic chiasm, obliterating the suprasellar cystern was found on MRI. Visuel field was normal. Octreotide LAR $20 \mathrm{mg} / \mathrm{month}$ and propranolol $40 \mathrm{mg} /$ day were started. After the 3 months treatment period, her laboratory tests were normal as shown in table-1. Pituitary MRI showed a $9 \times 6 \times 11 \mathrm{~mm}$ macroadenoma and a regression in diameter and compression of optic chiasm. Reoperation has been planned.

\section{CONCLUSION}

This case emphasizes that all pituitary adenomas should be screened for secretion of all hormones and immunohistochemical staining of all pituitary hormones is mandatory for correct classification.

Table-1:Laboratory results of patient before and after Octreotide LAR 20 $\mathrm{mg} / 28$ days treatment of 3 months period.

\begin{tabular}{|l|c|c|}
\hline & Basal & 3 months after treatment \\
\hline TSH (n=0,27-4,2 ulU/ml) & $2,3 \mathrm{ulU} / \mathrm{ml}$ & $1,55 \mathrm{ulU} / \mathrm{ml}$ \\
\hline $\mathrm{fT4}(\mathrm{n}=0,72-1,56 \mathrm{ng} / \mathrm{dl})$ & $1,66 \mathrm{ng} / \mathrm{dl}$ & $1,17 \mathrm{ng} / \mathrm{dl}$ \\
\hline $\mathrm{fT3}(\mathrm{n}=1,96-4,36 \mathrm{pg} / \mathrm{ml})$ & $4,38 \mathrm{pg} / \mathrm{ml}$ & $2,94 \mathrm{pg} / \mathrm{ml}$ \\
\hline $\mathrm{GH}(\mathrm{ng} / \mathrm{ml})$ & $2,09 \mathrm{ng} / \mathrm{ml}$ & $0,54 \mathrm{ng} / \mathrm{ml}$ \\
\hline IGF-1 $(\mathrm{n}=109-284 \mathrm{ng} / \mathrm{ml})$ & $205 \mathrm{ng} / \mathrm{ml}$ & $155 \mathrm{ng} / \mathrm{ml}$ \\
\hline Prolactin $(\mathrm{n}=6-29,9 \mathrm{ng} / \mathrm{dl})$ & $60 \mathrm{ng} / \mathrm{dl}$ & $14 \mathrm{ng} / \mathrm{dl}$ \\
\hline$\alpha$-glycoprotein subunit $(\mathrm{n}=0-0,9$ IU/L) & $0,74 \mathrm{IU} / \mathrm{L}$ & $0,45 \mathrm{IU} / \mathrm{L}$ \\
\hline SHBG & $97.1 \mathrm{nmol} / \mathrm{L}$ & $55,1 \mathrm{nmol} / \mathrm{L}$ \\
\hline$\alpha-$ glycoprotein subunit/TSH molar ratio & 3,2 & 2,9 \\
\hline
\end{tabular}

Table-2: Results of TRH (400 mcg) stimulation test

\begin{tabular}{|l|c|}
\hline TRH (400 mcg) STIMULATION TEST & TSH (ulU/ml) \\
\hline 0. $\mathrm{min}$ & 2,6 \\
\hline $20 \cdot \mathrm{min}$ & 3 \\
\hline $40 \cdot \mathrm{min}$ & 3,18 \\
\hline $60 \cdot \mathrm{min}$ & 3,02 \\
\hline $90 \cdot \mathrm{min}$ & 3,38 \\
\hline
\end{tabular}
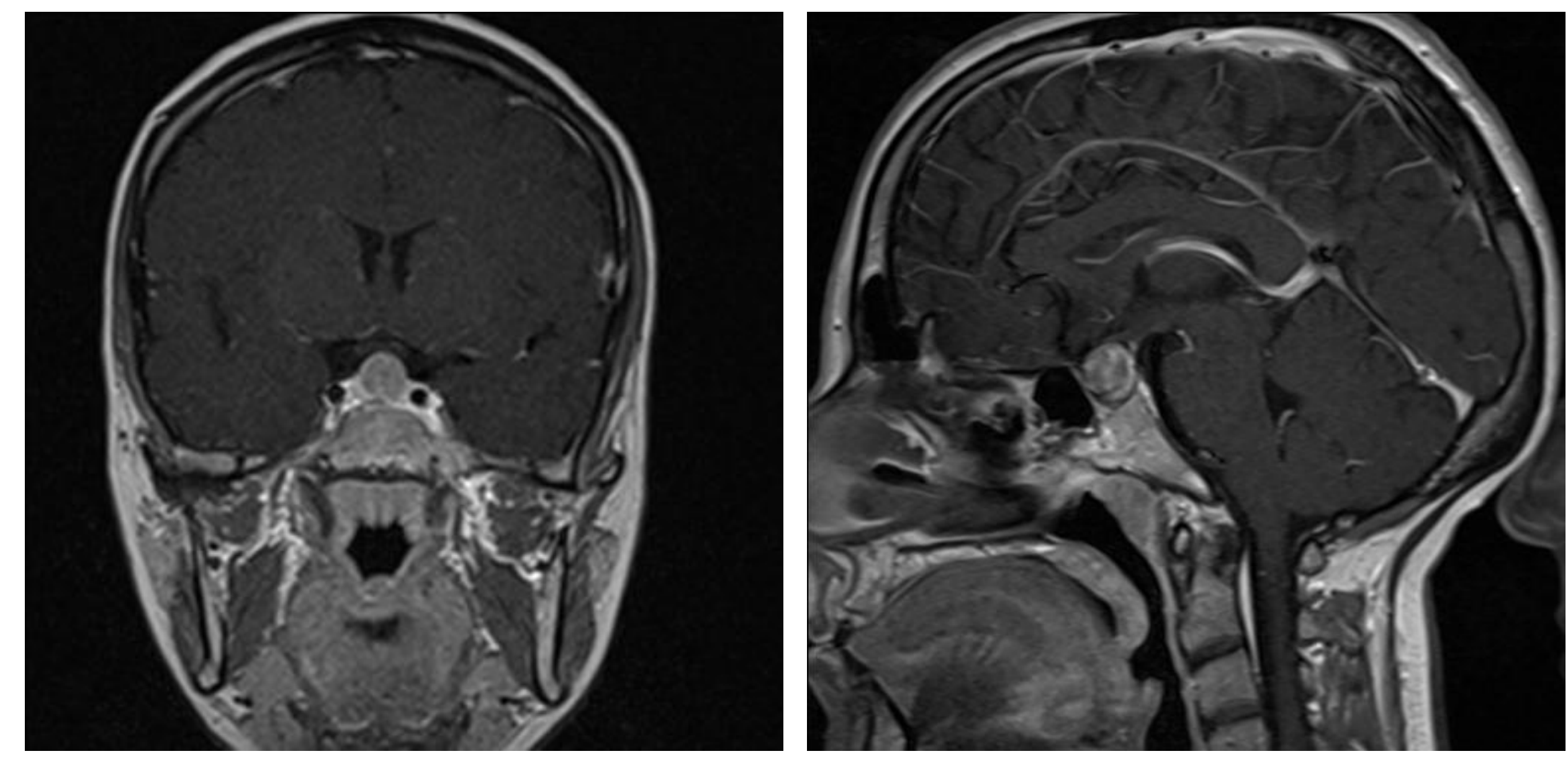

Figure-1: Postcontrast T1 weighted MRI after her pregnancy : coronal and sagittal sections visualising pituitary macroadenoma $(12 \times 11 \mathrm{~mm})$ with compression to optic chiasm and obliterating suprasellar cystern.
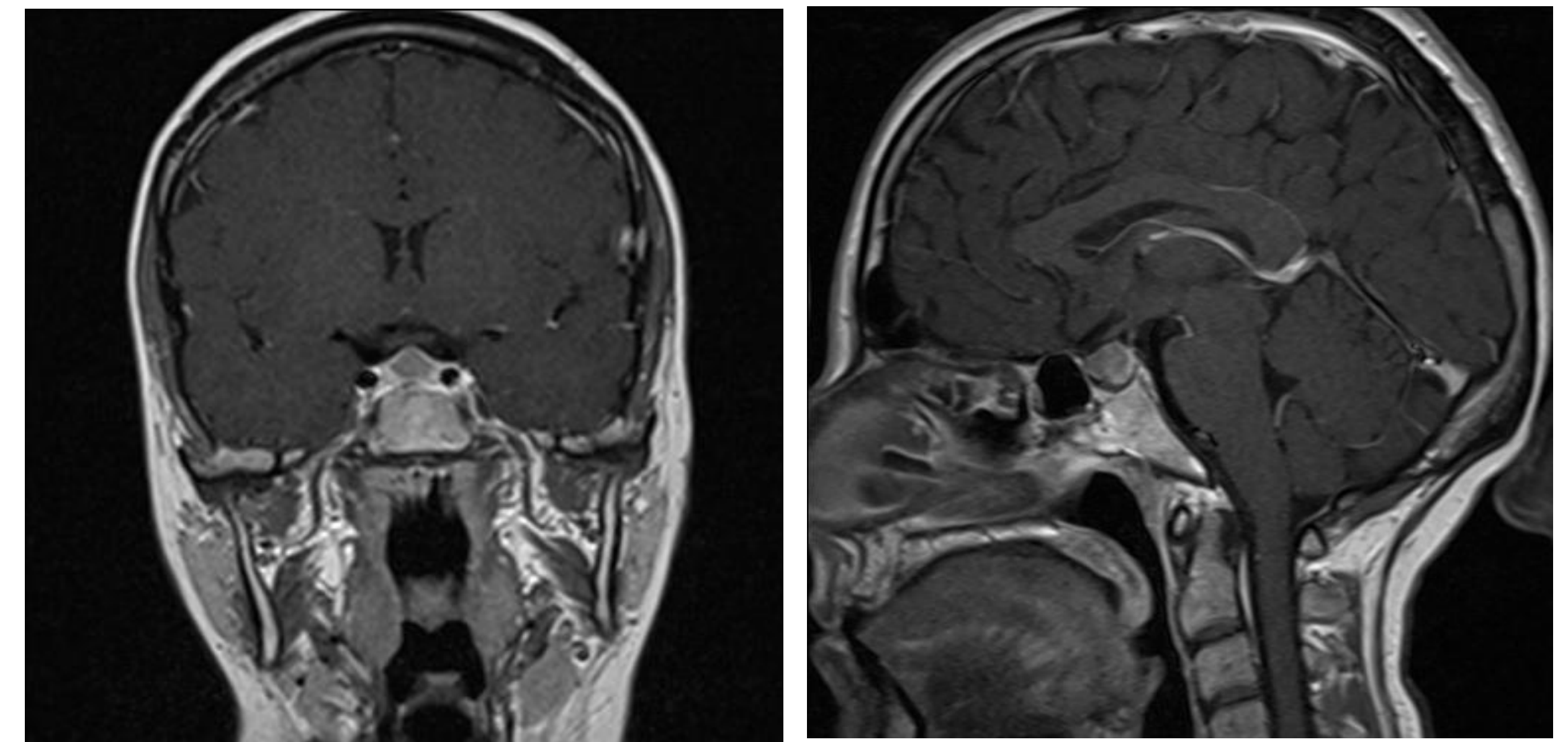

Figure-2: Postcontrast T1 weighted MRI after 3 months of Octreotide LAR $20 \mathrm{mg} / 28$ days treatment: coronal and sagittal sections visualising pituitary adenoma of $9 \times 6 \times 11 \mathrm{~mm}$ with regression in diameter and compression to optic chiasm. 